ENCYCLOPEEDIE Encyclopédie berbère

BERBERE

9 | 1991

9 | Baal - Ben Yasla

\title{
Ben Boulaïd Mostefa
}

(1917-1955)

\section{J. Morizot}

\section{OpenEdition}

\section{Journals}

Édition électronique

URL : http://journals.openedition.org/encyclopedieberbere/1672

DOI : $10.4000 /$ encyclopedieberbere. 1672

ISSN : 2262-7197

\section{Éditeur}

Peeters Publishers

\section{Édition imprimée}

Date de publication : 1 avril 1991

Pagination : 1441-1444

ISBN : 2-85744-509-1

ISSN : $1015-7344$

\section{Référence électronique}

J. Morizot, «Ben Boulaïd Mostefa », Encyclopédie berbère [En ligne], 9 | 1991, document B58, mis en ligne le 01 avril 2013, consulté le 24 septembre 2020. URL : http://journals.openedition.org/ encyclopedieberbere/1672; DOI : https://doi.org/10.4000/encyclopedieberbere.1672

Ce document a été généré automatiquement le 24 septembre 2020.

(c) Tous droits réservés 


\section{Ben Boulaiid Mostefa}

(1917-1955)

\section{J. Morizot}

1 Ben Boulaid Mostefa est l'un des «neuf chefs historiques" de la guerre pour l'indépendance de l'Algérie et, le premier novembre 1954, le responsable de la wilaya des Aurès-Nemencha dans le sud-constantinois. On le dit né à Arris, ce qui est vrai administrativement, mais ne veut rien dire. En effet, Arris aujourd'hui ville de plus de vingt mille habitants, siège de la daïra du même nom, ne regroupe en 1917 que les bureaux de la commune mixe de l'Aurès et les habitations de ses agents; en tout environ 75 personnes.

2 La famille Ben Boulaid appartient à la tribu berbérophone des Ouled Daoud, plus connue sous le nom de Touaba, la plus nombreuse et la plus puissante du massif aurasien ; son territoire s'étire sur plus de $70 \mathrm{~km}$ du nord-est au sud-ouest dans l'axe de la vallée de l'oued El-Abiod, confinant d'un côté avec Timgad, de l'autre avec l'oasis de Biskra. Jusqu'au début de ce siècle, les Touaba, entre lesquels s'est maintenue une très forte solidarité qui a résisté à la création des trois douars issus de leur démembrement, ont mené une vie très particulière, partagée entre la culture sèche en altitude, la culture irriguée dans les périmètres irrigables et l'élevage des chèvres et des moutons sur toute l'étendue de leur terroir, ce qui leur a imposé des déplacements saisonniers, l'utilisation de la tente pour habitat et la construction de greniers familiaux où ils déposaient leurs provisions et leurs biens et auprès desquels se situaient leurs lieux de prières et leurs cimetières.

3 Quand naît Mostefa Ben Boulaid, la vie des Touaba est en pleine évolution. Ayant maintenu leurs activités agricoles et en grande partie abandonné leurs activités pastorales, ils ont commencé à se sédentariser et à se construire des maisons en pierre auprès des anciens greniers familiaux. C'est ainsi qu'Inerkeb, l'ancien grenier des Ben Boulaid, est devenu son village, sa dechra.

Ben Boulaid appartient à ces familles dont l'évolution vers la sédentarité a été favorisée par le voisinage d'Arris, son développement et l'ouverture d'un certain nombre de commerces. Devenus sédentaires, les Touaba ont néanmoins conservé l'habitude de déplacements lointains nécessités par les soins à donner à leurs champs et à leurs 
jardins. Ils continuent d'aller et venir et connaissent parfaitement la montagne. Cela leur servira au jour de l'insurrection. Par ailleurs ce sont des gens fiers qui n'ont pas oublié les spoliations dont ils ont été les victimes en 1879: à cette époque leurs meilleures terres leur ont été enlevées et la blessure est restée ouverte.

Leur existence nomade explique leur refus de l'école du temps des Pères Blancs (1893-1916). Leurs dispositions ont complètement changé quand ils se sont fixés et l'on voit le jeune Ben Boulaid entrer à l'école primaire à Batna et non à Arris : probablement parce qu'il n'existe pas encore d'école au siège de la commune mixte ou qu'à ses débuts celle-ci est réservée aux enfants des agents communaux. Mostefa obtient le certificat d'études, ce qui est alors le plus haut niveau d'études que puisse atteindre un jeune rural et ce qui prouve qu'il maîtrise parfaitement le français. Sans doute aide-t-il alors son père dans ses activités, diverses comme celles de tous les montagnards qui ont toujours des terres à cultiver, quelques bêtes à élever. Puis vient le temps du service militaire auquel les Algériens se sont trouvés soumis dans les années qui précédèrent la première guerre mondiale et qu'ils ont fini par très bien accepter. Ben Boulaid fait son temps au $11^{\mathrm{e}}$ régiment de tirailleurs où il est bientôt nommé caporal. Rendu à la vie civile, il crée une petite entreprise de transports. Quand éclate la deuxième guerre mondiale en 1939, il a 22 ans; il est mobilisé, il participe aux combats et il aurait été blessé. En 1943 il est rappelé et ses états de service lors de la campagne d'Italie lui valent le grade d'adjudant, la croix de guerre et la médaille militaire.

6 L'administration locale apprécie cette homme "sérieux, honnête, réservé " et "d'apparence timide » et à son retour dans ses foyers, elle lui attribue, faveur insigne, une licence pour l'exploitation d'une ligne de cars de Batna à Arris et, dans cette période de pénurie où il a fallu réglementer le commerce des tissus, elle lui confie la présidence du groupement des commerçants chargés d'en faire la distribution, ce qui va lui permettre de se constituer tout un réseau de relations dans les douars. Pourtant on n'ignore pas tout à fait qu'il a eu un passé politique; il a adhéré au «Mouvement pour le triomphe des libertés démocratiques ", M.T.L.D., de Hadj Messali et il a même fait partie de son noyau dur, l'organisation spéciale, l'O.S. ; mais le chef de la commune mixte croit l'avoir ramené à de meilleurs sentiments.

7 En 1948, il se dévoile en se présentant comme candidat du M.T.L.D. aux élections à l'Assemblée Algérienne. Malgré toutes les pressions qui s'exercent sur les populations, il obtient 10000 voix au premier tour. C'est un succès considérable qui ne dissimuleront pas les résultats proclamés au second tour qui feront d'un individu méprisable (cf. Yves Courrière, Les Fils de la Toussaint, p. 128) l'élu officiel des Aurasiens. Pour le chef de la commune mixte qui s'est toujours flatté de tenir en mains les montagnards, c'est un aveu d'impuissance qui ne sera pas sans suite.

8 La disgrâce dans laquelle va tomber Mostefa Ben Boulaid, auquel est retiré sa carte violette, ne fait que renforcer son engagement politique ; il va feindre d'être découragé et de vouloir désormais se consacrer à ses affaires qui prospèrent. En réalité, il va déployer dans la clandestinité une activité considérable au service de la cause nationaliste. Le rôle qu'il joue à la tête du Parti a été reconnu en juillet 1951 avec son admission au Comité central mais ce qui semble lui importer avant tout c'est le renforcement de son autorité dans l'Aurès.

9 Habile politique, il parviendra à s'assurer l'appui précieux de petits groupes d'individus qui ont pris le maquis, dans la tradition demeurée bien vivante dans les mémoires de ces « bandits d'honneur » qui, à la fin de la guerre mondiale, ont tenu en échec pendant 
des mois les troupes lancées à leur poursuite. Il a réussi en outre à réconcilier les gens de sa tribu, les Touaba, et ceux de la tribu des Beni Bou Slimane, ennemis de toujours.

En juillet 1951, en dépit de la présence d'un administrateur et de gendarmes, ses partisans parviennent à empêcher le déroulement des élections dans un douar de la commune mixte. En 1952, l'insécurité s'y est développée à un tel point que le gouvernement général a estimé urgent d'éloigner le chef de la commune mixte en place depuis dix ans. Pour établir l'autorité de son successeur une opération militaire de grande envergure est menée : c'est la manœuvre «Aiguillé » qui, à partir du 15 août 1952, a mis en action plusieurs milliers d'hommes contre « les bandits de l'Aurès » car, à l'époque, on ne voit dans les troubles qu'une manifestation du banditisme traditionnel.

11 Échec complet et nouvel aveu d'impuissance, la manœuvre "Aiguille " montre à Mostefa Ben Boulaid qu'avec quelques hommes de plus, un peu plus d'argent, un peu plus d'armes, il est capable de se mesurer avec l'armée française dont l'essentiel des forces est engagé dans la guerre d'Indochine.

Des armes, il ne lui est pas difficile d'en trouver, les troupes de l'Axe en ont laissé des quantités dans leur fuite et, depuis, les Stati italiens et les Mauser allemands font l'objet d'un actif commerce de contrebande sur les confins tuniso-lybiens.

Il a deux ans devant lui pour parfaire son organisation et faire partager aux autres ses espérances ; mais il ne parviendra à convaincre ni Hadj Messali qu'il ira voir à Niort, ni les «Centralistes" qui se sont séparés de ce dernier. Les uns et les autres estiment qu'engager le combat dans l'état des forces révolutionnaires, c'est « envoyer le peuple à l'abattoir ». Ben Boulaid se retrouve seulement avec quatre "frères » pour créer le "Comité révolutionnaire d'unité et d'action » ou C.R.U.A.

Si Ben Boulaid estime pouvoir se passer des politiques, en revanche il attache un grand prix à l'appui des Kabyles. Il ira donc voir Krim Belkaceme et finira au moins par l'ébranler car Krim ne rompra pas totalement avec Hadj Messali et, le $1^{\text {er }}$ novembre, les Kabyles resteront dans l'expectative.

15 Le 25 juillet 1954, ils ne seront que 22 autour de lui et certains d'entreeux n'iront pas jusqu'au bout de leur engagement.

16 Le $1^{\text {er }}$ novembre Ben Boulaid sera donc à peu près seul avec seulement ses Aurasiens, 359 hommes bien armés, d'après les chiffres que l'on connaîtra après son arrestation. Et ce sera la surprise, car depuis la manđEuvre « Aiguille », il aura soigneusement évité de faire quoi que ce soit qui puisse alerter les autorités. Surprise aussi pour les habitants de l'Aurès qui apprendront presque tous par la voie des journaux que la montagne est en insurrection.

17 Tous les effectifs dont pouvait disposer la $11^{\mathrm{e}}$ région militaire seront envoyés sur place, y compris légionnaires et tabors marocains, sans beaucoup plus de résultats qu'en 1952 mais avec des effets beaucoup plus traumatisants pour les Aurasiens.

18 Ben Boulaid sera arrêté en février 1955 à la frontière tuniso-lybienne par des éléments français, au retour d'un voyage qu'il avait entrepris pour se procurer des armes. Les papiers saisis sur lui confirmèrent qu'il était bien le chef de l'insurrection dans l'Aurès et révélèrent tous les détails de l'organisation qu'il avait mise sur pied; mais c'est seulement beaucoup plus tard que fut connu son rôle au niveau « national ».

19 Jugé et condamné à mort, Ben Boulaid s'évadera le 4 novembre 1955 et regagnera le massif où depuis son arrestation régnait l'anarchie. Sa mort, le 27 mars 1956, à la suite 
de la manipulation d'un colis piégé priva l'Aurès de la seule tête capable de réaliser l'union des montagnards.

\section{BIBLIOGRAPHIE}

BIRONNEAU R., Correspondance avec l'auteur.

BITAT Rabah, Comment nous avons préparé le $1^{\mathrm{er}}$ novembre 1954, Journal l'Express, 3 novembre 1979.

CAZEBONNE G., Correspondance avec l'auteur.

COURRIÈRE Y., La guerre d'Algérie, t. 1 ; Les Fils de la Toussaint, A. Fayard, 1968.

HARBI Mohammed, Le F.L.N. mirage et réalité, éditions J.A., 1980.

LE MOUDJAHID, Commémoration du $23^{\mathrm{e}}$ anniversaire de la mort de Mostefa Ben Boulaid, 25 mars 1979

VAUJOUR J., De la révolte à la révolution, Albin Michel, 1985.

INDEX

Mots-clés : Biographie 